\title{
Amplitude Modulation and Synchronization of Fractional-Order Memristor-Based Chua's Circuit
}

\author{
A. G. Radwan, ${ }^{1,2}$ K. Moaddy, ${ }^{3}$ and I. Hashim ${ }^{4}$ \\ ${ }^{1}$ Department of Engineering Mathematics and Physics, Faculty of Engineering, Cairo University, Giza 12613, Egypt \\ ${ }^{2}$ NISC Research Center, Nile University, Egypt \\ ${ }^{3}$ Department of Mathematics, Faculty of Science and Arts, Shaqra University, Saudi Arabia \\ ${ }^{4}$ School of Mathematical Sciences, Universiti Kebangsaan Malaysia, 43600 Bangi, Selangor, Malaysia \\ Correspondence should be addressed to A. G. Radwan; agradwan@ieee.org
}

Received 19 July 2013; Accepted 14 October 2013

Academic Editor: Bashir Ahmad

Copyright (c) 2013 A. G. Radwan et al. This is an open access article distributed under the Creative Commons Attribution License, which permits unrestricted use, distribution, and reproduction in any medium, provided the original work is properly cited.

\begin{abstract}
This paper presents a general synchronization technique and an amplitude modulation of chaotic generators. Conventional synchronization and antisynchronization are considered a very narrow subset from the proposed technique where the scale between the output response and the input response can be controlled via control functions and this scale may be either constant (positive, negative) or time dependent. The concept of the proposed technique is based on the nonlinear control theory and Lyapunov stability theory. The nonlinear controller is designed to ensure the stability and convergence of the proposed synchronization scheme. This technique is applied on the synchronization of two identical fractional-order Chua's circuit systems with memristor. Different examples are studied numerically with different system parameters, different orders, and with five alternative cases where the scaling functions are chosen to be positive/negative and constant/dynamic which covers all possible cases from conventional synchronization to the amplitude modulation cases to validate the proposed concept.
\end{abstract}

\section{Introduction}

Despite that the history of fractional calculus started in the same period of time as integer calculus, the major revolutions in this area have been discovered only during the last five decades where the realization, modeling, and numerical simulations were available [1-4]. Similarly, the chaotic systems have been studied heavily during the last four decades since they play an important role in industrial applications particularly in chemical reactions [5], biological systems [6], circuit theory [7-11], control [12], and security applications [13-16]. Recently much attention has been devoted to the search for better and more efficient methods for the control or determination of a solution, approximate or analytical, of chaotic systems.

Antisynchronization is a phenomenon in which the state vectors of the synchronized systems have the same amplitude but opposite signs to those of the driving system. Therefore, the sum of two signals is expected to converge to zero when antisynchronization appears. Since the discovery of antisynchronization experimentally in the context of selfsynchronization, it has been applied in many different fields, such as biological and physical systems, structural engineering, and ecological models [17]. Liu et al. [18] show that either synchronization or antisynchronization can appear depending on the initial conditions of the coupled pendula. Active control method is used to study the antisynchronization for two identical and nonidentical systems [19-21].

The synchronization of fractional chaotic systems has started to attract much attention and has also raised up some problems [22-24]. Recently the consistency of the improvement of models based on fractional-order differential structure has increased in reputation in the research of dynamical systems [25-27]. Yu et al. studied the synchronization of three chaotic fractional-order Lorenz systems with bidirectional coupling [28], Odibat et al. [29] investigated the chaos synchronization of two identical systems via linear control, and Bhalekar and Daftardar-Gejji [30] demonstrated that two different fractional-order chaotic systems can be synchronized using active control. 
Recently, Radwan et al. [31] developed a framework to obtain approximate numerical solutions of the fractionalorder Chua's circuit with memristor using a nonstandard finite difference method. The most important advantage of using fractional order is to increase the chaotic range as proven from the stability analyses of this circuit in both the W-plane and s-plane. Moreover, another circuit from Chua's family was studied and modified with the use of memristor with detailed responses by Petráš in [32]. In this paper, the concept of dynamic scaling between the master and slave systems in the fractional-order domain is introduced. The proposed concept covers the conventional synchronization up to the amplitude modulation technique. Different examples have been studied based on the nonlinear antisynchronization of two identical fractional-order chaotic Chua's circuits with memristor. This generalized projective synchronization is based on the nonlinear control theory and Lyapunov stability theory.

The paper is organized as follows. In Section 2 we provide some mathematical models of the fractional calculus theory and describe the nonstandard finite difference scheme to solve fractional differential equations and in the last subsection we present the fractional Chua's circuit with memristor. Nonlinear antisynchronization of two identical fractionalorder chaotic Chua's circuit systems is also introduced in Section 3, while the generalized projective synchronization is discussed in Section 4. Finally, conclusions are drawn in the last section.

\section{Fractional Order System Memristor Based Chua's Circuit}

Recently, most of the dynamical systems based on the integerorder calculus have been modified into the fractional order domain due to the extra degrees of freedom which increase the flexibility of the design and can be used to precisely fit the experimental data much better than the integer-order modeling. The Caputo fractional derivative of order $\alpha$ of a continuous function $f: R^{+} \rightarrow R$ is defined as follows:

$$
\begin{aligned}
& D^{\alpha} f(t) \\
& \quad \equiv \frac{d^{\alpha} f(t)}{d t^{\alpha}} \\
& \quad= \begin{cases}\frac{1}{\Gamma(m-\alpha)} \int_{0}^{t} \frac{f^{(m)}(\tau)}{(t-\tau)^{\alpha-m+1}} d \tau, & m-1<\alpha<m, \\
\frac{d^{m}}{d t^{m}} f(t), & \alpha=m,\end{cases}
\end{aligned}
$$

where $m$ is the first integer greater than $\alpha$ and $\Gamma(\cdot)$ is the gamma function and is defined by

$$
\Gamma(z)=\int_{0}^{\infty} e^{-t} t^{z-1} d t, \quad \Gamma(z+1)=z \Gamma(z) .
$$

The Grünwald-Letnikov method of approximation for the one-dimensional fractional derivative is as follows [33]:

$$
\begin{gathered}
D^{\alpha} x(t)=f(t, x), \\
D^{\alpha} x(t)=\lim _{h \rightarrow 0} h^{-\alpha} \sum_{j=0}^{t / h}(-1)^{j}\left(\begin{array}{c}
\alpha \\
j
\end{array}\right) x(t-j h),
\end{gathered}
$$

where $\alpha>0$ and $D^{\alpha}$ denotes the fractional derivative. Also $N=[t / h]$, and $h$ is the step size. Therefore, (3a) and (3b) is discretized as follows:

$$
\sum_{j=0}^{n+1} c_{j}^{\alpha} x(t-j h)=f\left(t_{n}, x\left(t_{n}\right)\right), \quad n=1,2,3, \ldots,
$$

where $t_{n}=n h$ and $c_{j}^{\alpha}$ are the Grünwald-Letnikov coefficients defined as

$$
\begin{gathered}
c_{j}^{\alpha}=\left(1-\frac{1+\alpha}{j}\right) c_{j-1}^{\alpha}, \\
c_{0}^{\alpha}=h^{-\alpha}, \quad j=1,2,3, \ldots .
\end{gathered}
$$

The nonstandard discretization technique [34] is a general scheme where we replace the step size $h$ by a function $\varphi(h)$. By applying this technique and using the Grünwald-Letnikov discretization method $[2,31]$, it yields the following relations:

$$
x_{n+1}=\frac{-\sum_{j=1}^{n+1} c_{j}^{\alpha} x_{n+1-j}+f_{1}\left(t_{n+1}, x_{n+1}\right)}{c_{0}^{\alpha_{1}}}
$$

where $c_{0}^{\alpha_{1}}=\left(\varphi_{1}(h)\right)^{-1}$ are functions of the step size $h=\Delta t$, with $\varphi_{1}(h)=h+O\left(h^{2}\right)$, when $h \rightarrow 0$. The fractional-order Chua's circuit [31] based on the new nanodevice element which is called memristor [35-38] is shown in Figure 1 with its $q-\phi$ relationship. The canonical memristor-based fractionalorder Chua's circuit can be written as follows:

$$
\begin{gathered}
\left(\begin{array}{c}
D^{\alpha} x \\
D^{\beta} y \\
D^{\gamma} z \\
D u
\end{array}\right) \\
=\left(\begin{array}{cccc}
-a k & a & 0 & 0 \\
-1 & 0 & 1 & 0 \\
0 & -b & c & 0 \\
1 & 0 & 0 & 0
\end{array}\right)\left(\begin{array}{l}
x \\
y \\
z \\
u
\end{array}\right), \\
k= \begin{cases}m_{1} & |u|<1, \\
m_{2} & |u|>1,\end{cases}
\end{gathered}
$$

where $a, b, c, m_{1}$, and $m_{2}$ are related to the circuit parameters [31]. The effect of the memristive property appears through the parameter $k$ (the slope of the charge with respect to the flux in the memristor) which is a piecewise-linear function with two different slopes $m_{1}$ and $m_{2}$ depending on the value of the flux $u$ (Figure 1(b)). It is clear that the system has four variables which are $\{x, y, z$, and $u\}$ and the chaotic behavior exists in a wider range as proved in [31]. The chaotic behavior has been verified by the calculation of the maximum 


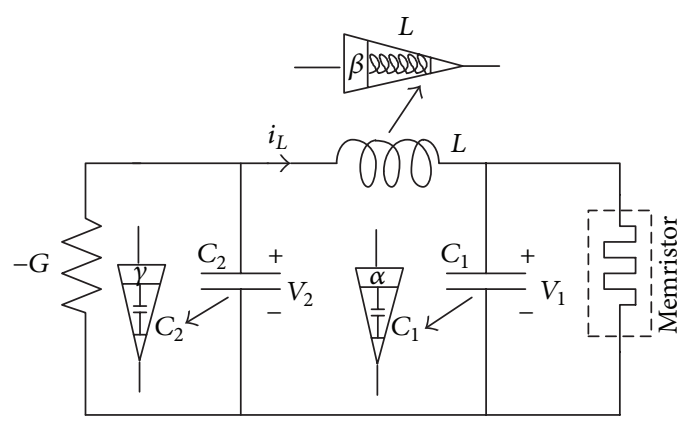

(a)

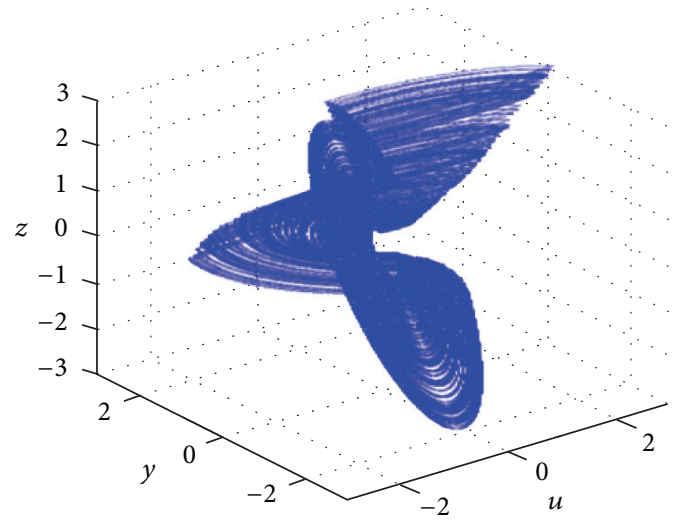

(c)

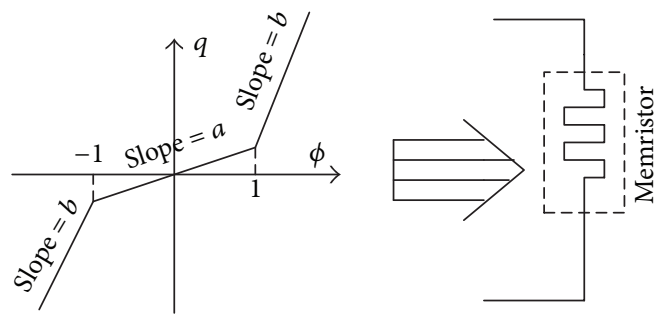

(b)

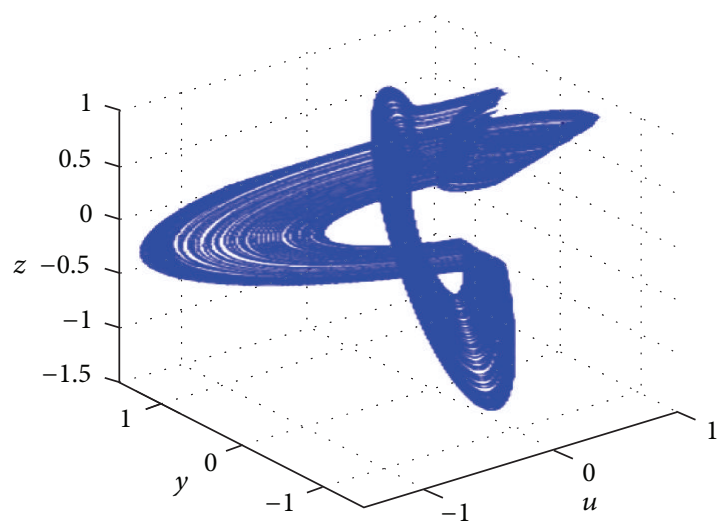

(d)

Figure 1: (a) The canonical Chua's circuit with memristor, (b) the piecewise-linear flux-controlled memristor, and (c) 3D shapes in the $u-y$ - $z$ plane when $(\alpha, \beta, \gamma)(\mathrm{a})(1.0,0.9,0.9)$ and $(\mathrm{d})(1.1,0.9,0.9)$.

Lyapunov exponent (MLE). Figures 1(c) and 1(d) show the chaotic responses for two different cases where the $\alpha$ order changes from 1.0 to 1.1. It is clear from these subfigures that the output response is very sensitive to the fractional-order parameters. Therefore, these fractional-order parameters can be used for extra degrees of freedom and also to control the behavior of the strange attractors of the Chua's circuit.

\section{The Proposed Technique}

From the previous section, the fractional-order memristorbased Chua's circuit has ten parameters which are three fractional-orders $\{\alpha, \beta, \gamma\}$, three system parameters $\{a, b, c\}$, and the initial conditions. Figure 2 shows the general block diagram of the proposed system assuming that the fractionalorders of the two systems are identical but the other seven parameters may be different. The idea is based on modifying the equations of the second system by adding control functions $\left\{u_{1}, u_{2}, u_{3}, u_{4}\right\}$ on the four equations to minimize the functions $x_{1}+s_{1} x_{2}, y_{1}+s_{2} y_{2}, z_{1}+s_{3} z_{2}$, and $w_{1}+s_{4} w_{2}$ to approach zero. In this case, the output response of the second system is given by

$$
\begin{aligned}
& x_{2}(t)=-\frac{x_{1}(t)}{s_{1}(t)}, \\
& y_{2}(t)=-\frac{y_{1}(t)}{s_{2}(t)},
\end{aligned}
$$

$$
\begin{aligned}
& z_{2}(t)=-\frac{z_{1}(t)}{s_{3}(t)}, \\
& w_{2}(t)=-\frac{w_{1}(t)}{s_{4}(t)} .
\end{aligned}
$$

Then, if $s_{1}=s_{2}=s_{3}=s_{4}=-1$, this will be the conventional synchronization, and if $s_{1}=s_{2}=s_{3}=s_{4}=1$, this will be the antisynchronization as will be discussed in the next subsection. Moreover, the scaling functions $s_{i}(t), i=1,2,3,4$, can have different values and time-dependent functions are discussed in the next subsections.

3.1. Nonlinear Antisynchronization of Two Identical Fractional-Order Chaotic Chua's Circuit Systems $\left(s_{i}=1, i=\right.$ $1,2,3,4)$. In this subsection we observe the antisynchronization behavior in two identical fractional-order chaotic Chua's circuit systems via nonlinear control. The drive (master) and slave (response) systems are described, respectively, by the following equations. However, the initial condition on the drive system is different from that of the response system:

$$
\begin{gathered}
D^{\alpha} x_{1}(t)=a_{1}\left(y_{1}-f\left(w_{1}\right) x_{1}\right), \\
D^{\beta} y_{1}(t)=z_{1}-x_{1},
\end{gathered}
$$




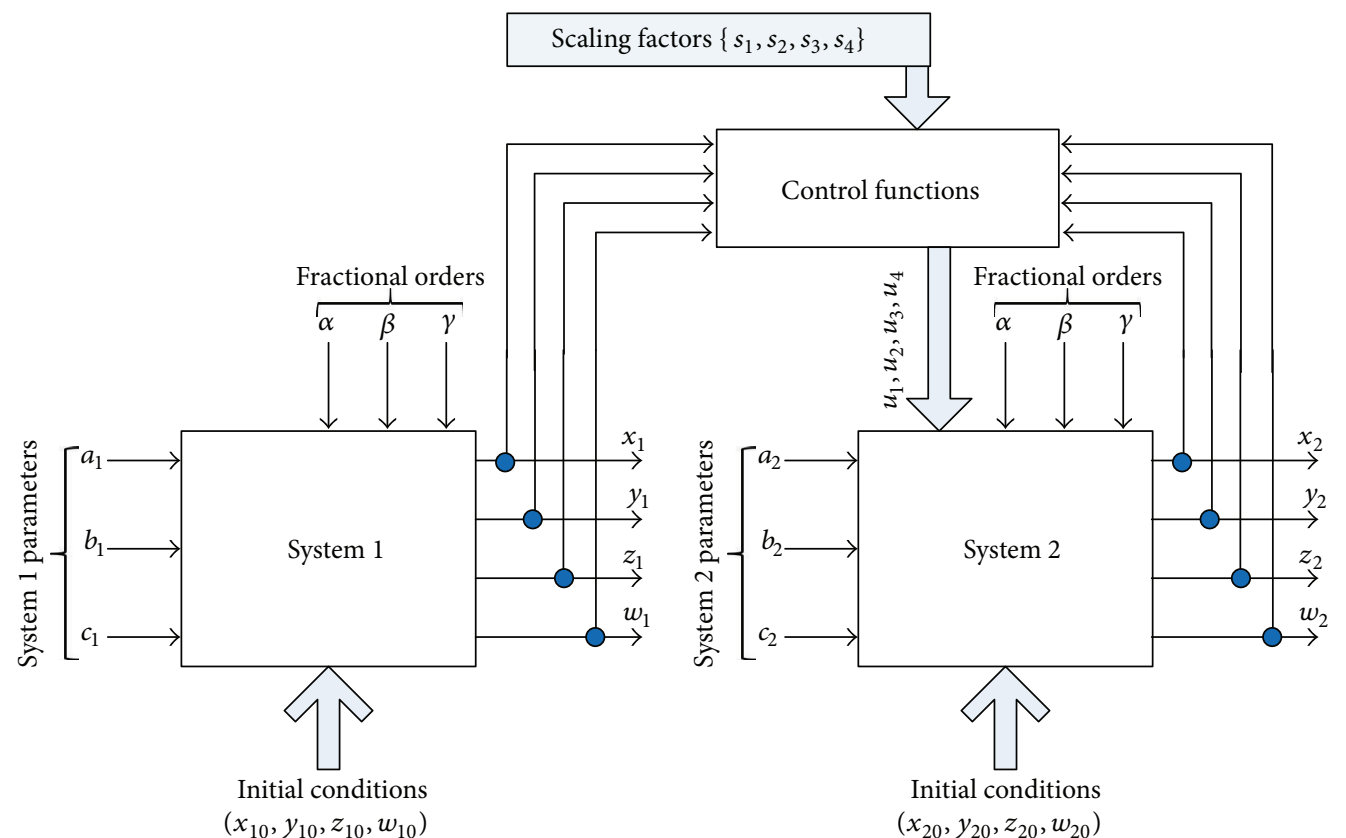

Figure 2: The proposed block diagram.

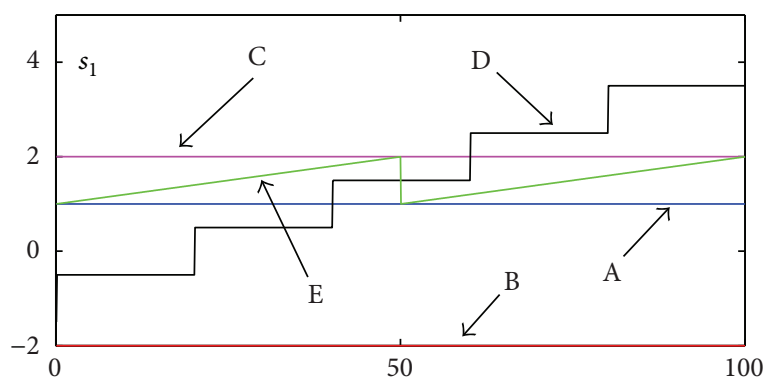

(a)

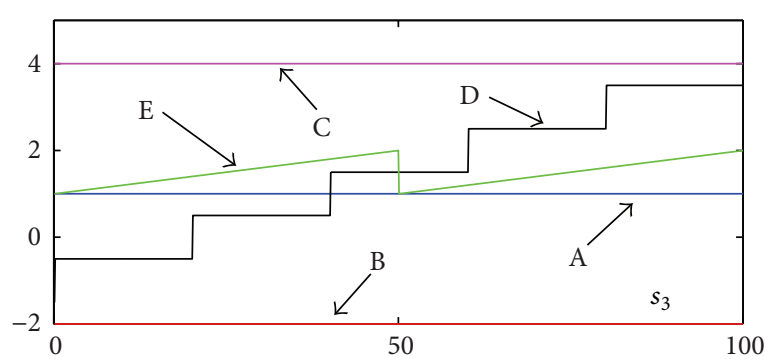

(c)

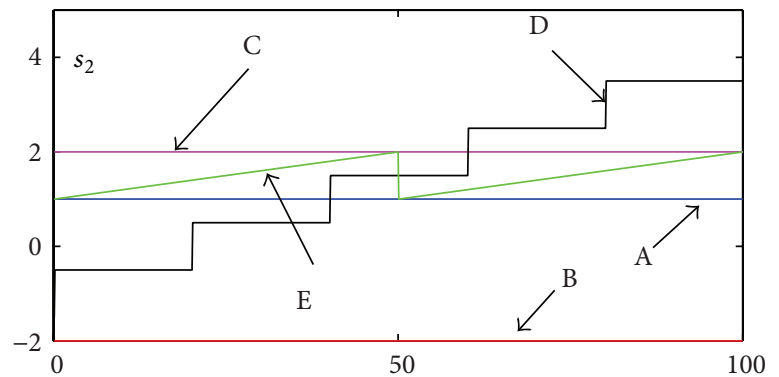

(b)

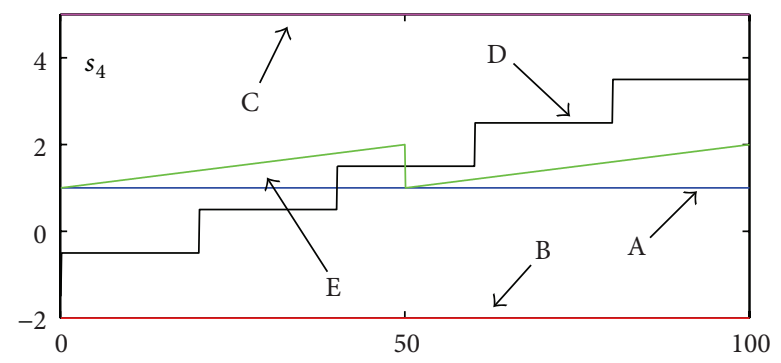

(d)

Figure 3: The five different cases of the parameters $s_{1}, s_{2}, s_{3}$, and $s_{4}$ as function of time where Case A; $\left\{s_{1}, s_{2}, s_{3}, s_{4}\right\}=\{1,1,1,1\}$ Case B; $\left\{s_{1}, s_{2}, s_{3}, s_{4}\right\}=\{-2,-2,-2,-2\}$ Case C; $\left\{s_{1}, s_{2}, s_{3}, s_{4}\right\}=\{2,3,4,5\}$ Case D; $s_{1}=s_{2}=s_{3}=s_{4}=-0.5+$ integer part of $(t / 20)$, Case E; $s_{1}=s_{2}=s_{3}=s_{4}=1+($ fraction part of $(t / 50)) / 50$.

$$
\begin{gathered}
D^{\gamma} z_{1}(t)=-b_{1} y_{1}+c_{1} z_{1}, \\
D w_{1}(t)=x_{1}, \\
D^{\alpha} x_{2}(t)=a_{2}\left(y_{2}-f\left(w_{2}\right) x_{2}\right)+u_{1}, \\
D^{\beta} y_{2}(t)=z_{2}-x_{2}+u_{2},
\end{gathered}
$$

$$
\begin{gathered}
D^{\gamma} z_{2}(t)=-b_{2} y_{2}+c_{2} z_{2}+u_{3}, \\
D w_{2}(t)=x_{2}+u_{4},
\end{gathered}
$$

where the control function $u=\left[u_{1} ; u_{2} ; u_{3} ; u_{4}\right]^{T}=0$. Our goal is to determine the control function to make the first derivative of $V(e)$, that is, $V(e)<0$. From (9a), (9b), (9c), 


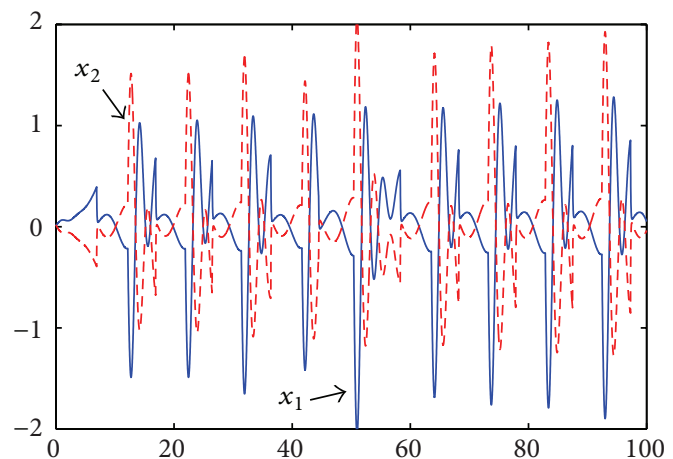

(a)

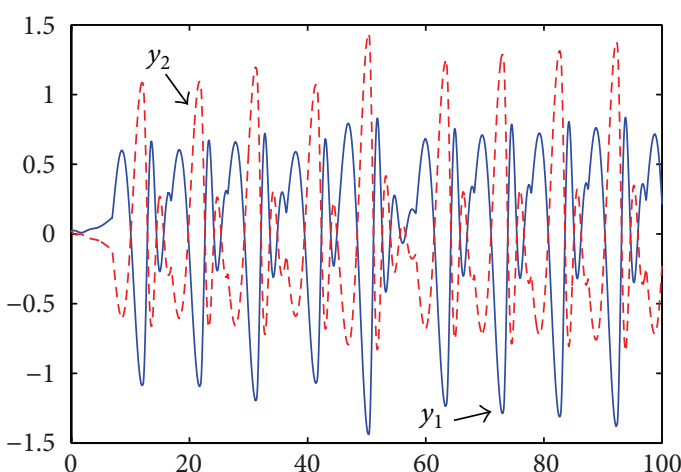

(b)

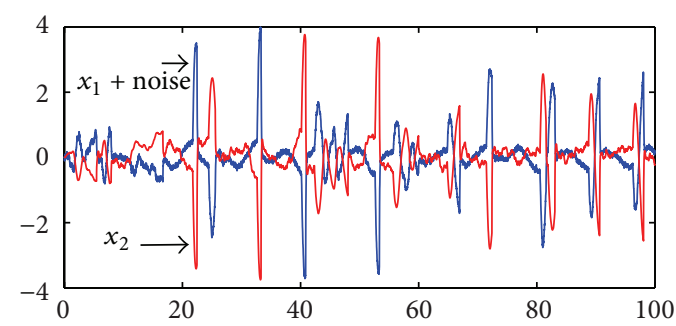

(c)

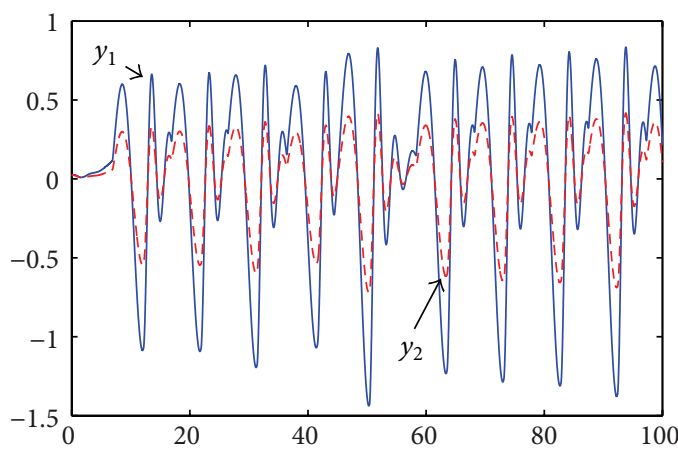

(d)

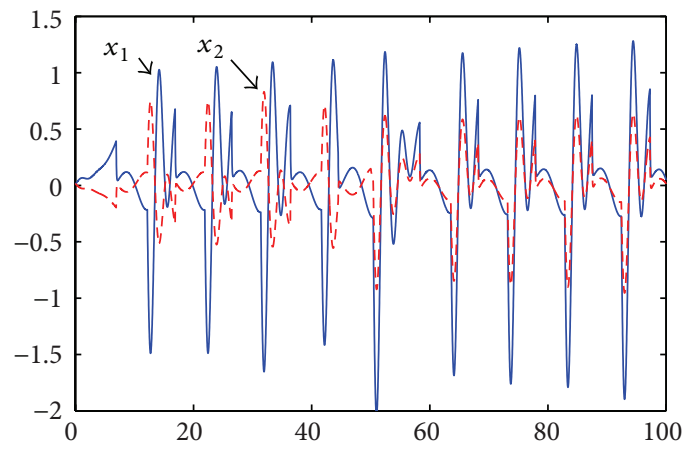

(e)

FiguRE 4: The general synchronization of (a), (b) the $x$ and $y$ responses when $\left\{\alpha, \beta, \gamma, a_{1}, b_{1}, c_{1}, a_{2}, b_{2}, c_{2}\right\}=\{0.96,0.96,0.96,4,1,0.65,4,1,0.65\}$ for Case $\mathrm{A}$, (c) the $x$ response under the same condition with additive noise with amplitude 0.1 , (d) the response when $\left\{\alpha, \beta, \gamma, a_{1}, b_{1}, c_{1}, a_{2}, b_{2}, c_{2}\right\}=\{0.96,0.96,0.96,4,1,0.65,4,1,0.65\}$ for Case B, and (e) Case C.

and (9d) to (10a), (10b), (10c), and (10d), the error equations when the system parameters $\left\{a_{1}, b_{1}, c_{1}\right\}=\left\{a_{2}, b_{2}, c_{2}\right\}=\{a, b, c\}$ are given as follows:

$$
\begin{gathered}
D^{\alpha} e_{x}=a e_{y}-a\left(f\left(w_{1}\right) x_{1}-f\left(w_{2}\right) x_{2}\right)+u_{1}, \\
D^{\beta} e_{y}=e_{z}-e_{x}+u_{2} \\
D^{\gamma} e_{z}=-b e_{y}+c e_{z}+u_{3}, \\
D e_{w}=e_{x}+u_{4}
\end{gathered}
$$

where $e_{x}=x_{2}+x_{1}, e_{y}=y_{2}+y_{1}, e_{z}=z_{2}+z_{1}, e_{w}=w_{2}+w_{1}$. In order to determine the controller, let

$$
u_{1}=u_{1 a}+u_{1 b}, \quad u_{1 b}=-a\left(f\left(w_{1}\right) x_{1}+f\left(w_{2}\right) x_{2}\right)
$$

Then we rewrite system (11a), (11b), (11c), and (11d) in the following form:

$$
\begin{gathered}
D^{\alpha} e_{x}=a e_{y}+u_{1 b}, \\
D^{\beta} e_{y}=e_{z}-e_{x}+u_{2}, \\
D^{\gamma} e_{z}=-b e_{y}+c e_{z}+u_{3}, \\
D e_{w}=e_{x}+u_{4} .
\end{gathered}
$$

Based on the Lyapunov stability theory, when the controller satisfies the assumption $V(e)=0.5 e^{T} e$, a positive definite function, the first derivative of $V(e), V(e)<0$, and the antisynchronization of two identical Chua's circuit systems 
from different initial conditions is achieved, we choose $u$ as follows:

$$
\begin{gathered}
u_{1 b}=-e_{x}-a e_{y}, \\
u_{2}=-e_{z}+e_{x}-e_{y}, \\
u_{3}=-2 c e_{z}+b e_{y}, \\
u_{4}=-e_{x}-e_{u} .
\end{gathered}
$$

Theorem 1. The nonlinear controller achieves global antisynchronization between two identical Chua's circuit systems master (9a), (9b), (9c), and (9d) and slave (10a), (10b), (10c), and $(10 \mathrm{~d})$.

Proof. Take a Lyapunov function for (13a), (13b), (13c), and (13d) into consideration as

$$
V(e)=0.5 e^{T} e .
$$

We then get the first derivative of $V(e)$ :

$$
\begin{aligned}
\dot{V}= & e_{x}\left(a e_{y}+u_{1 b}\right)+e_{y}\left(e_{z}-e_{x}+u_{2}\right) \\
& +e_{z}\left(-b e_{y}+c e_{z}+u_{3}\right)
\end{aligned}
$$

$$
\begin{gathered}
+e_{w}\left(e_{x}+u_{4}\right), \\
\dot{V}=-e_{x}^{2}-e_{y}^{2}-b c e_{z}^{2}-e_{w}^{2},
\end{gathered}
$$

so $\dot{V}(e)<0$ is satisfied. Since $\dot{V}(e)$ is a negative-definite function [39], the error states tend to zero at steady state. Therefore, the states of controlled response system and drive system are globally antisynchronized asymptotically.

3.2. Generalized Projective Synchronization of Two Identical Chua's Circuits. In this subsection we study the synchronization between two identical fractional-order chaotic Chua's circuits based on the generalized projective synchronization. The unknown terms $u_{1}, u_{2}, u_{3}$, and $u_{4}$ in (11a), (11b), (11c), and (11d) are active control functions to be determined. Define the error functions as

$$
\left(\begin{array}{c}
e_{x}(t) \\
e_{y}(t) \\
e_{z}(t) \\
e_{w}(t)
\end{array}\right)=\left(\begin{array}{c}
x_{1}(t)+s_{1}(t) x_{2}(t) \\
y_{1}(t)+s_{2}(t) y_{2}(t) \\
z_{1}(t)+s_{3}(t) z_{2}(t) \\
w_{1}(t)+s_{4}(t) w_{2}(t)
\end{array}\right)
$$

Then by similar procedure, the control functions are given by

$$
\left(\begin{array}{l}
u_{1}(t) \\
u_{2}(t) \\
u_{3}(t) \\
u_{4}(t)
\end{array}\right)=\left(\begin{array}{c}
\frac{\left(a_{1} f\left(w_{1}(t)\right)-k_{x}\right) x_{1}(t)-a_{1} y_{1}(t)}{s_{1}(t)}+\left(a_{2} f\left(w_{2}(t)\right)-k_{x}\right) x_{2}(t)-a_{2} y_{2}(t) \\
\frac{x_{1}(t)-z_{1}(t)-k_{y} y_{1}(t)}{s_{2}(t)}+x_{2}(t)-z_{2}(t)-k_{y} y_{2}(t) \\
\frac{b_{1} y_{1}(t)-c_{1} z_{1}(t)-k_{z} z_{1}(t)}{s_{3}(t)}+b_{2} y_{2}(t)-c_{2} z_{2}(t)-k_{z} z_{2}(t) \\
\frac{-x_{1}(t)-k_{w} w_{1}(t)}{s_{4}(t)}+-x_{2}(t)-k_{w} w_{2}(t)
\end{array}\right)
$$

Then

$$
\begin{aligned}
& \left(\begin{array}{c}
D^{\alpha} e_{x} \\
D^{\beta} e_{y} \\
D^{\gamma} e_{z} \\
D e_{w}
\end{array}\right) \\
& =\left(\begin{array}{cccc}
-k_{x} & 0 & 0 & 0 \\
0 & -k_{y} & 0 & 0 \\
0 & 0 & -k_{z} & 0 \\
0 & 0 & 0 & -k_{w}
\end{array}\right)\left(\begin{array}{l}
e_{x} \\
e_{y} \\
e_{z} \\
e_{w}
\end{array}\right)
\end{aligned}
$$

which are decaying functions as the values of $k_{x}, k_{y}, k_{z}$, and $k_{w}$ are positive.

\section{Numerical Results}

Nonstandard finite difference method with $\phi(h)=1-e^{-h}$ is used to solve the systems of differential equations. In addition, a time step size 0.01 is employed. We select the parameters of the master system of Chua's circuit system as $a_{1}=4, b_{1}=1, c_{1}=0.65, m_{1}=0.2$, and $m_{2}=5$, so these systems exhibit chaotic behavior with the following initial conditions:

$$
\begin{aligned}
& \left(\begin{array}{l}
x_{1}(0) \\
y_{1}(0) \\
z_{1}(0) \\
w_{1}(0)
\end{array}\right)=\left(\begin{array}{l}
0.01 \\
0.02 \\
0.01 \\
0.05
\end{array}\right), \\
& \left(\begin{array}{l}
x_{2}(0) \\
y_{2}(0) \\
z_{2}(0) \\
w_{2}(0)
\end{array}\right)=\left(\begin{array}{l}
0.02 \\
0.03 \\
0.02 \\
0.06
\end{array}\right) .
\end{aligned}
$$

To validate the proposed technique, five different cases of the scaling parameters $s_{1}(t), s_{2}(t), s_{3}(t)$, and $s_{4}(t)$ are discussed as shown in Figure 3 and we have the following.

(i) Case $A\left(\left\{s_{1}, s_{2}, s_{3}, s_{4}\right\}=\{1,1,1,1\}\right)$. In this case the scaling parameters are positive ones; then the output responses of system 2 are the antisynchronization of the system 1 output, that is, $x_{2}=-x_{1}$ and the same for other outputs 


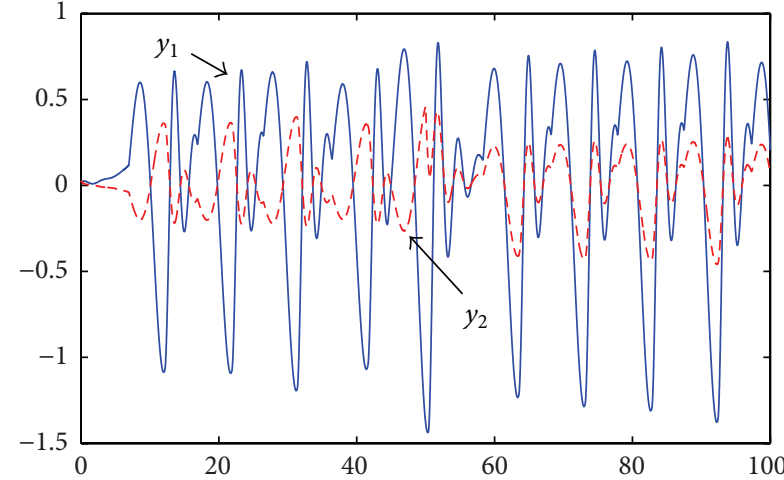

(a)

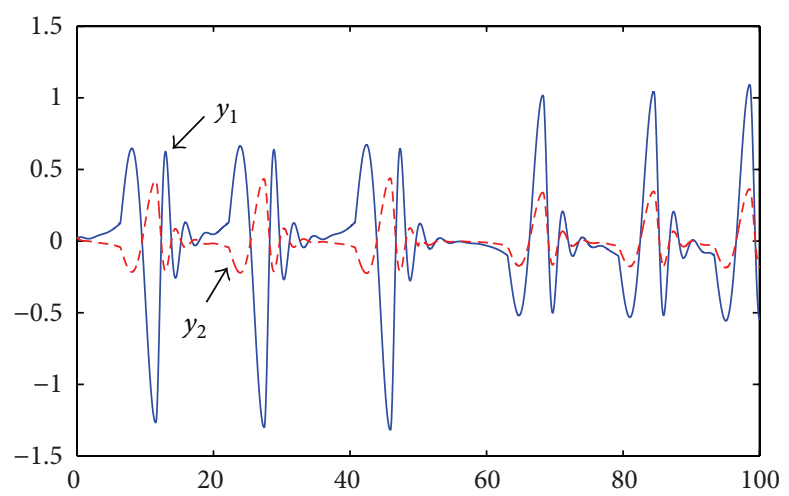

(c)

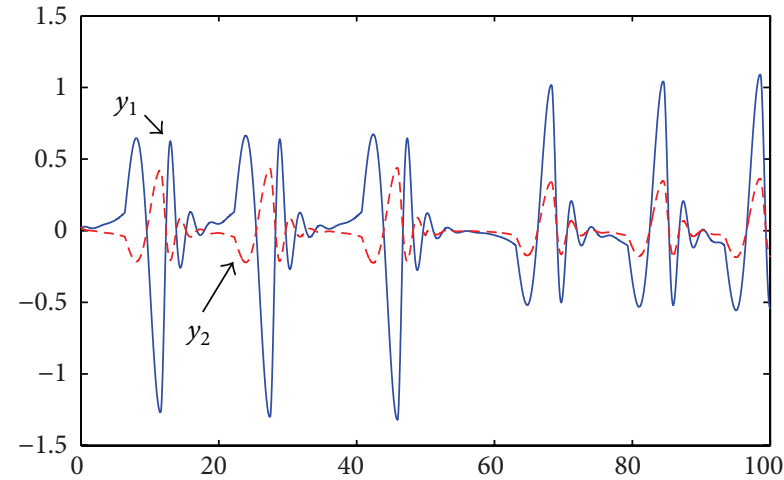

(b)

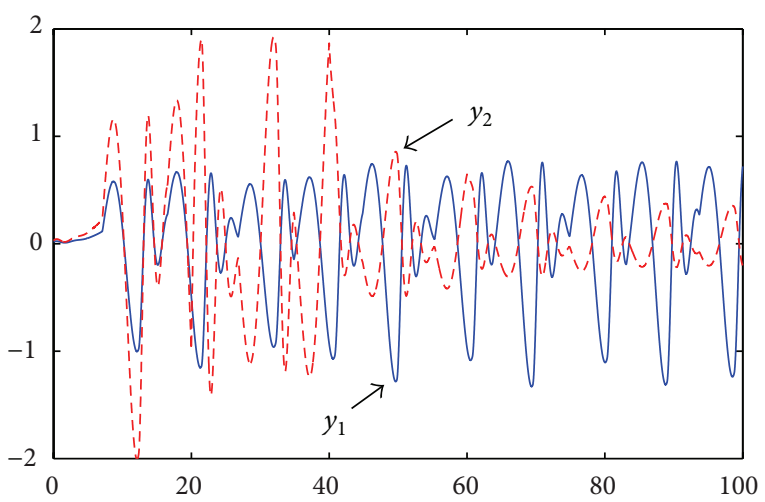

(d)

Figure 5: The $y$ response when for Case D when (a) $\left\{\alpha, \beta, \gamma, a_{1}, b_{1}, c_{1}, a_{2}, b_{2}, c_{2}\right\}=\{0.96,0.96,0.96,4,1,0.65,4,1,0.65\},(\mathrm{b})\left\{\alpha, \beta, \gamma, a_{1}\right.$, $\left.b_{1}, c_{1}, a_{2}, b_{2}, c_{2}\right\}=\{0.9,0.95,1,4,1,0.65,4,1,0.65\}$, (c) $\left\{\alpha, \beta, \gamma, a_{1}, b_{1}, c_{1}, a_{2}, b_{2}, c_{2}\right\}=\{0.9,0.95,1,4,1,0.65,4.5,1.2,0.55\}$, and $(\mathrm{d})\left\{\alpha, \beta, \gamma, a_{1}, b_{1}\right.$, $\left.c_{1}, a_{2}, b_{2}, c_{2}\right\}=\{0.95,0.95,0.95,4,1,0.65,4,1,0.65\}$.

(see Figures 4(a) and 4(b)). Moreover, when an additive random noise is added to both the $X_{1}$ and $Y_{1}$ with amplitude 0.1 under the same conditions, the output response tries to follow the changes as shown in Figure 4(c).

(ii) Case $B\left(\left\{s_{1}, s_{2}, s_{3}, s_{4}\right\}=\{-2,-2,-2,-2\}\right)$. In this case the scaling parameters are equal and negative; then the output responses of system 2 are the half-synchronization of the system 1 output, that is, $x_{2}=0.5 x_{1}$ and the same for other outputs (see Figure $4(\mathrm{~d})$ ).

(iii) Case $C\left(\left\{s_{1}, s_{2}, s_{3}, s_{4}\right\}=\{2,3,4,5\}\right)$. In this case the scaling parameters are different constants and positive; then the output responses of system 2 have different antisynchronization scale than system 1 output, that is, $x_{2}=-0.5 x_{1}, y_{2}=-y_{1} / 3$, $z_{2}=-z_{1} / 4$, and $w_{2}=-w_{1} / 5$ (see Figure $4(\mathrm{e})$ ).

(iv) Case $D\left(s_{1}=s_{2}=s_{3}=s_{4}=-0.5+\right.$ integer part of $\left.(t / 20)\right)$. In this case the scaling parameters are time dependent (staircase) as steps (every 20 seconds); then the output responses of system 2 will start with double synchronization in the first 20 seconds and then double antisynchronization in the following 20 seconds, and the scale factor increases with time every 20 seconds (see Figure 5). (v) Case $E\left(s_{1}=s_{2}=s_{3}=s_{4}=1+\right.$ (fraction part) of $(t / 50)) / 50$. In this case the scaling parameters are time dependent as they ramp up to 50 seconds and then reset and increase again. Since it is always positive, then the output response will be the antisynchronization of system 1 with a different scale (see Figure 6).

Figure 5 shows the proposed technique under different orders and different system parameters where the output will follow the expected response; however the transient time until the synchronization happens may be increased. Figures 5(b) and 5(c) illustrate the response with different fractional orders where $\alpha=0.9, \beta=0.95$, and $\gamma=1.0$, and Figure 5(c) shows the responses for different fractional orders and also system parameters $\left\{\alpha, \beta, \gamma, a_{1}, b_{1}, c_{1}, a_{2}, b_{2}, c_{2}\right\}=$ $\{0.9,0.95,1,4,1,0.65,4.5,1.2,0.55\}$. Moreover, Figure 5(d) shows the system responses for equal fractional orders and different system parameters only. Therefore, many cases have been discussed when the synchronization parameters are changed by steps showing great matching with the expected results. Figure 6 illustrates the projections and system responses when the synchronization parameters change gradually and linearly resulting in a sawtooth waveform (Case E). It is clear from the previous responses for different system parameters, fractional orders, and time-independent 


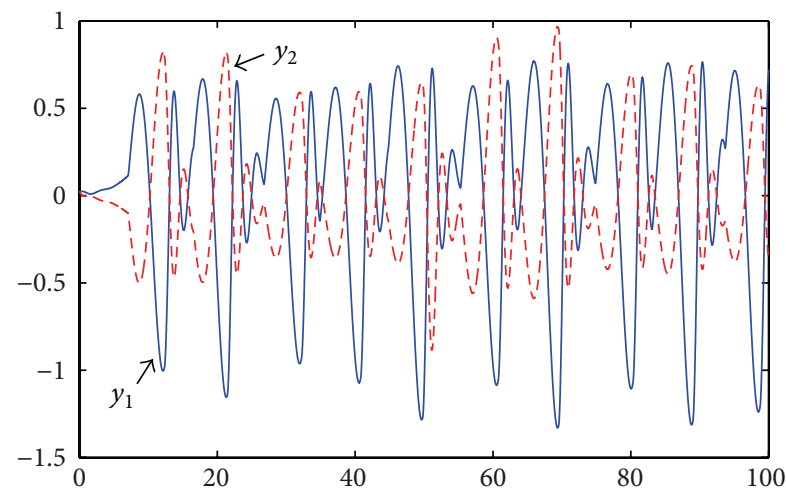

(a)

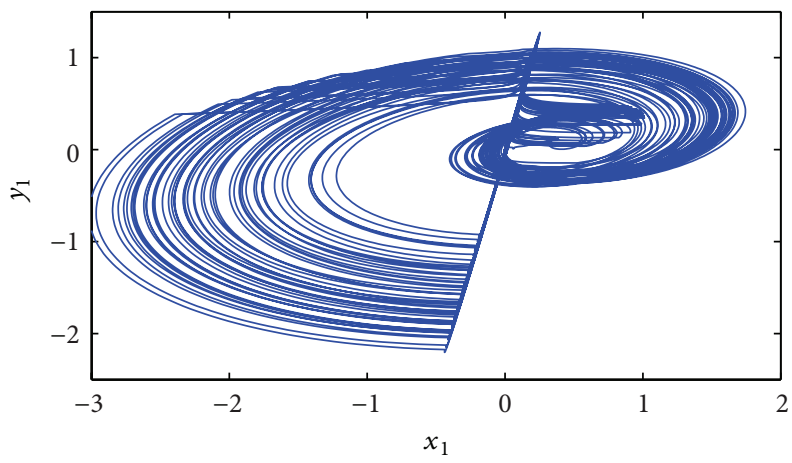

(c)

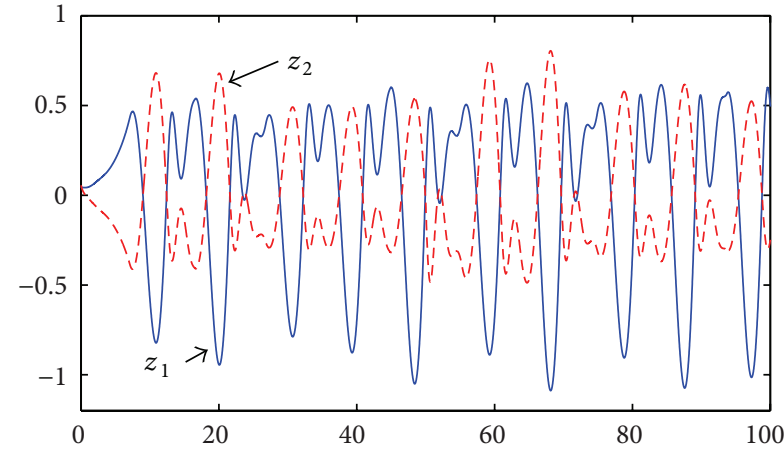

(b)

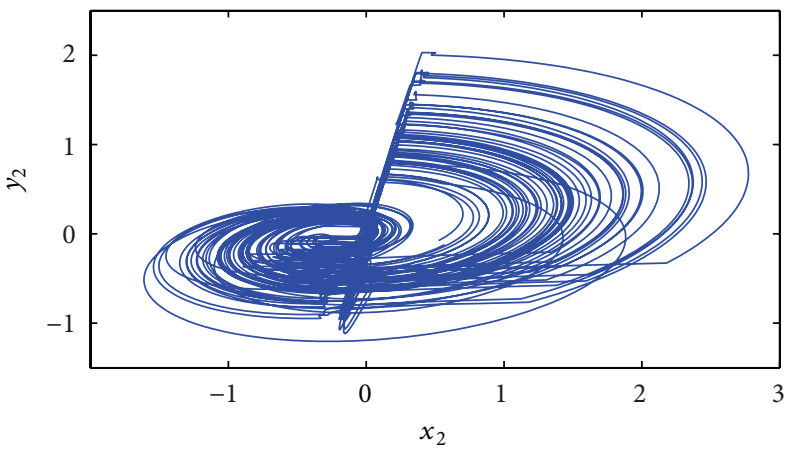

(d)

Figure 6: ((a), (b)) the $x_{1}-y_{1}$ and $x_{2}-y_{2}$ projections, and ((c), (d)) the $x, y$, and $z$ responses when $\left\{\alpha, \beta, \gamma, a_{1}, b_{1}, c_{1}, a_{2}, b_{2}, c_{2}\right\}=$ $\{0.95,0.95,0.95,4,1,0.65,4,1,0.65\}$ for Case $\mathrm{E}$.
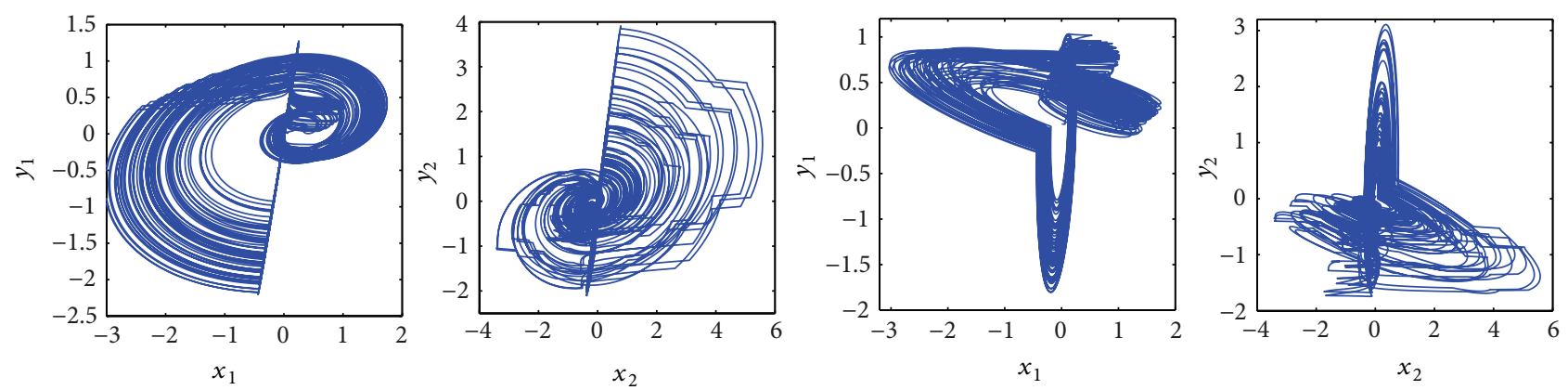

(a)

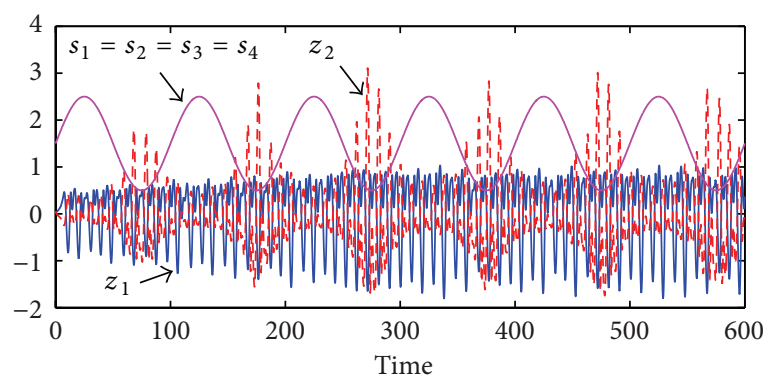

(b)

FIgURE 7: The projection and responses for the amplitude modulation when $s_{1}(t)=s_{2}(t)=s_{3}(t)=s_{4}(t)=1.5+\sin (0.02 \pi t)$. 
and time-dependent synchronization parameters that the proposed method has many advantages and can be used for the synchronization of any two chaotic systems. There are many recent research articles which discuss the importance of communication techniques using chaotic signals [40, 41]. In the amplitude modulation, the amplitude of the output signal should be a function of the input signal. In Figure 7, we assumed that the information data $s_{1}(t)=s_{2}(t)=s_{3}(t)=$ $s_{4}(t)=1.5+\sin (0.02 \pi t)$. Therefore, the output will be different from system 1 output as shown from the strange attractors shown in Figure 7(a). The time waveform of the scaling functions, $z_{1}(t)$, and $z_{2}(t)$, is shown in Figure 7(b) where the modulation is clear. The demodulation can be done similarly by reversing the operation.

\section{Conclusions}

This paper discussed the concept of a general time-dependent synchronization scheme based on a nonlinear controller and then applied this technique on the fractional-order Chua's circuit with memristor. This nonlinear controller is based on the control theory and Lyapunov stability theory to achieve the required synchronization. Many examples including antisynchronization, synchronization, and both (as in Case D) between two identical fractional-order Chua's circuits with same/different fractional orders and same/different system parameters are discussed using numerical simulations by the nonstandard finite method. This technique can be repeated for other chaotic systems in a similar way to achieve good results.

\section{Conflict of Interests}

The authors declare that there is no conflict of interests regarding the publication of this paper.

\section{References}

[1] I. Podlubny, Fractional Differential Equations, vol. 198, Academic Press, San Diego, Calif, USA, 1999.

[2] L. Dorcak, Numerical Models for the Simulation of the Fractional-Order Control Systems, Technical University of Kosice, Kosice, Slovakia, 1994.

[3] A. Babakhani and D. Baleanu, "Existence and uniqueness of solution for a class of nonlinear fractional order differential equations," Abstract and Applied Analysis, vol. 2012, Article ID 632681, 14 pages, 2012.

[4] T. Abdeljawad, B. Benli, and D. Baleanu, "A generalized $q$ mittag-leffler function by $q$-captuo fractional linear equations," Abstract and Applied Analysis, vol. 2012, Article ID 546062, 11 pages, 2012.

[5] S. K. Han, C. Kerrer, and Y. Kuramoto, "Dephasing and burstling in coupled neural oscillators," Physical Review Letters, vol. 75, pp. 3190-3193, 1995.

[6] K. Moaddy, A. G. Radwan, K. N. Salama, S. Momani, and I. Hashim, "The fractional-order modeling and synchronization of electrically coupled neuron systems," Computers \& Mathematics with Applications, vol. 64, no. 10, pp. 3329-3339, 2012.
[7] A. G. Radwan, A. S. Elwakil, and A. M. Soliman, "Fractionalorder sinusoidal oscillators: design procedure and practical examples," IEEE Transactions on Circuits and Systems I, vol. 55, no. 7, pp. 2051-2063, 2008.

[8] A. G. Radwan, A. M. Soliman, and A. S. Elwakil, "First-order filters generalized to the fractional domain," Journal of Circuits Systems \& Computers, vol. 17, pp. 55-66, 2008.

[9] A. Shamim, A. G. Radwan, and K. N. Salama, "Fractional smith chart theory," IEEE Microwave and Wireless Components Letters, vol. 21, no. 3, pp. 117-119, 2011.

[10] A. G. Radwan, "Stability analysis of the fractional-order RLC circuit," Journal of Fractional Calculus and Applications, vol. 3, no. 1, pp. 1-15, 2012.

[11] A. G. Radwan, "Resonance and quality factor of the fractional RLC circuit," IEEE Journal on Emerging and Selected Topics in Circuits and Systems, vol. 3, no. 3, pp. 377-385, 2013.

[12] A. G. Radwan, K. Moaddy, K. N. Salama, S. Momani, and I. Hashim, "Control and switching synchronization of fractional order chaotic systems using active control technique," Journal of Advanced Research, 2013.

[13] A. G. Radwan, S. K. Abd-El-Hafiz, and S. H. Abd-El-Haleem, "Image encryption in the fractional-order domain," in Proceedings of the International Conference on Engineering and Technology (ICET '12), 2012.

[14] M. Feki, "An adaptive chaos synchronization scheme applied to secure communication," Chaos, Solitons and Fractals, vol. 18, no. 1, pp. 141-148, 2003.

[15] K. Murali and M. Lakshmanan, "Secure communication using a compound signal from generalized synchronizable chaotic systems," Physics Letters A, vol. 241, no. 6, pp. 303-310, 1998.

[16] T. Yang, "A survey of chaotic secure communication systems," International Journal of Computational Cognition, vol. 2, no. 2, pp. 81-130, 2004.

[17] I. Wedekind and U. Parlitz, "Experimental observation of synchronization and anti-synchronization of chaotic lowfrequency-fluctuations in external cavity semiconductor lasers," International Journal of Bifurcation and Chaos in Applied Sciences and Engineering, vol. 11, no. 4, pp. 1141-1147, 2001.

[18] J.-b. Liu, C.-f. Ye, S.-j. Zhang, and W.-t. Song, "Anti-phase synchronization in coupled map lattices," Physics Letters A, vol. 274, no. 1-2, pp. 27-29, 2000.

[19] M.-C. Ho, Y.-C. Hung, and C.-H. Chou, "Phase and anti-phase synchronization of two chaotic systems by using active control," Physics Letters A, vol. 296, no. 1, pp. 43-48, 2002.

[20] G.-H. Li and S.-P. Zhou, "Anti-synchronization in different chaotic systems," Chaos, Solitons and Fractals, vol. 32, no. 2, pp. 516-520, 2007.

[21] S. Bhalekar and V. Daftardar-Gejji, "Antisynchronization of nonidentical fractional-order chaotic systems using active control," International Journal of Differential Equations, vol. 2011, Article ID 250763, 13 pages, 2011.

[22] L. M. Pecora and T. L. Carroll, "Synchronization in chaotic systems," Physical Review Letters, vol. 64, no. 8, pp. 821-824, 1990.

[23] L. M. Pecora and T. L. Carroll, "Driving systems with chaotic signals," Physical Review A, vol. 44, no. 4, pp. 2374-2383, 1991.

[24] D. Bin, W. Jiang, and F. Xiangyang, "Chaotic synchronization with gap junction of multi-neurons in external electrical stimulation," Chaos, Solitons and Fractals, vol. 25, no. 5, pp. 1185-1192, 2005. 
[25] J. Yan and C. Li, "On chaos synchronization of fractional differential equations," Chaos, Solitons \& Fractals, vol. 32, no. 2, pp. 725-735, 2007.

[26] L. O. Chua, M. Itoh, L. Kocarev, and K. Eckert, "Chaos synchronization in Chua's circuit," Journal of Circuits, Systems, and Computers, vol. 3, no. 1, pp. 93-108, 1993.

[27] C. Li and T. Zhou, "Synchronization in fractional-order differential systems," Physica D, vol. 212, no. 1-2, pp. 111-125, 2005.

[28] Y. Yu, H.-X. Li, and Y. Su, "The synchronization of three chaotic fractional-order Lorenz systems with bidirectional coupling," Journal of Physics, vol. 96, no. 1, Article ID 012113, 2008.

[29] Z. M. Odibat, N. Corson, M. A. Aziz-Alaoui, and C. Bertelle, "Synchronization of chaotic fractional-order systems via linear control," International Journal of Bifurcation and Chaos in Applied Sciences and Engineering, vol. 20, no. 1, pp. 81-97, 2010.

[30] S. Bhalekar and V. Daftardar-Gejji, "Synchronization of different fractional order chaotic systems using active control," Communications in Nonlinear Science and Numerical Simulation, vol. 15, pp. 3536-3546, 2010.

[31] A. G. Radwan, K. Moaddy, and S. Momani, "Stability and nonstandard finite difference method of the generalized Chua's circuit," Computers \& Mathematics with Applications, vol. 62, no. 3, pp. 961-970, 2011.

[32] I. Petráš, "Fractional-order memristor-based Chua's circuit," IEEE Transactions on Circuits and Systems II, vol. 57, no. 12, pp. 975-979, 2010.

[33] G. Hussian, M. Alnaser, and S. Momani, "Non-standard discretization of fractional differential equations," in Proceedings of the 8th seminar of differential equations and dynamical systems, 2008.

[34] Applications of Nonstandard Finite Difference Schemes, World Scientific Publishing, River Edge, NJ, USA, 2000.

[35] A. G. Radwan, M. A. Zidan, and K. N. Salama, "HP memristor mathematical model for periodic signals and DC," in Proceedings of the IEEE International Midwest Symposium on Circuits and Systems, pp. 861-864, 2010.

[36] A. Talukdar, A. G. Radwan, and K. N. Salama, "Non linear dynamics of memristor based 3rd order oscillatory system," Microelectronics Journal, vol. 43, no. 3, pp. 169-175, 2012.

[37] A. G. Radwan, M. A. Zidan, and K. N. Salama, "HP Memristor mathematical model for periodic signals and DC," in Proceedings of the 53rd IEEE International Midwest Symposium on Circuits and Systems (MWSCAS '10), pp. 861-864, Seattle, Wash, USA, August 2010.

[38] A. G. Radwan, M. A. Zidan, and K. N. Salama, "On the mathematical modeling of Memristors," in Proceedings of the International Conference on Microelectronics (ICM '10), pp. 284287, December 2010.

[39] W. Hahn, Stability of Motion, Springer, New York, NY, USA, 1967.

[40] G. Kolumbán, M. P. Kennedy, and L. O. Chua, "The role of synchronization in digital communications using chaos. II. Chaotic modulation and chaotic synchronization," IEEE Transactions on Circuits and Systems I, vol. 45, no. 11, pp. 11291140, 1998.

[41] N. J. Corron and D. W. Hahs, "A new approach to communications using chaotic signals," IEEE Transactions on Circuits and Systems I, vol. 44, no. 5, pp. 373-382, 1997. 


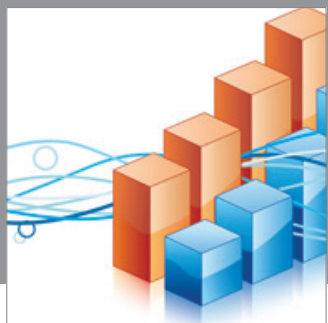

Advances in

Operations Research

mansans

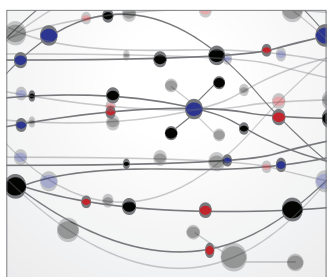

The Scientific World Journal
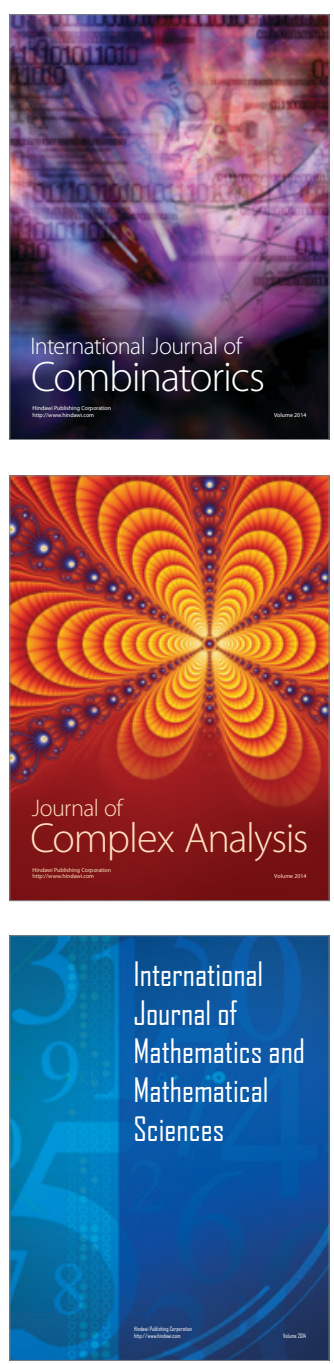
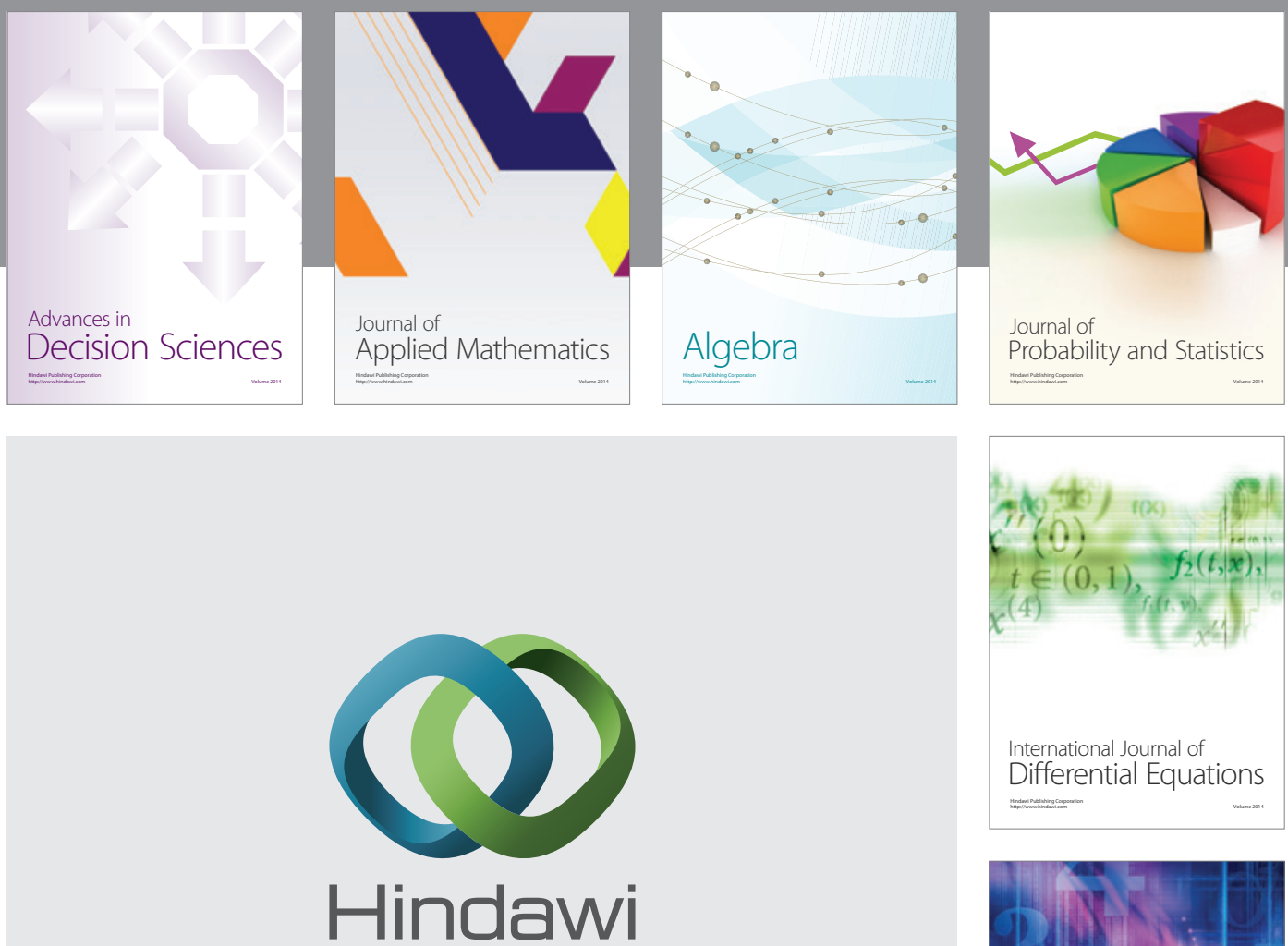

Submit your manuscripts at http://www.hindawi.com
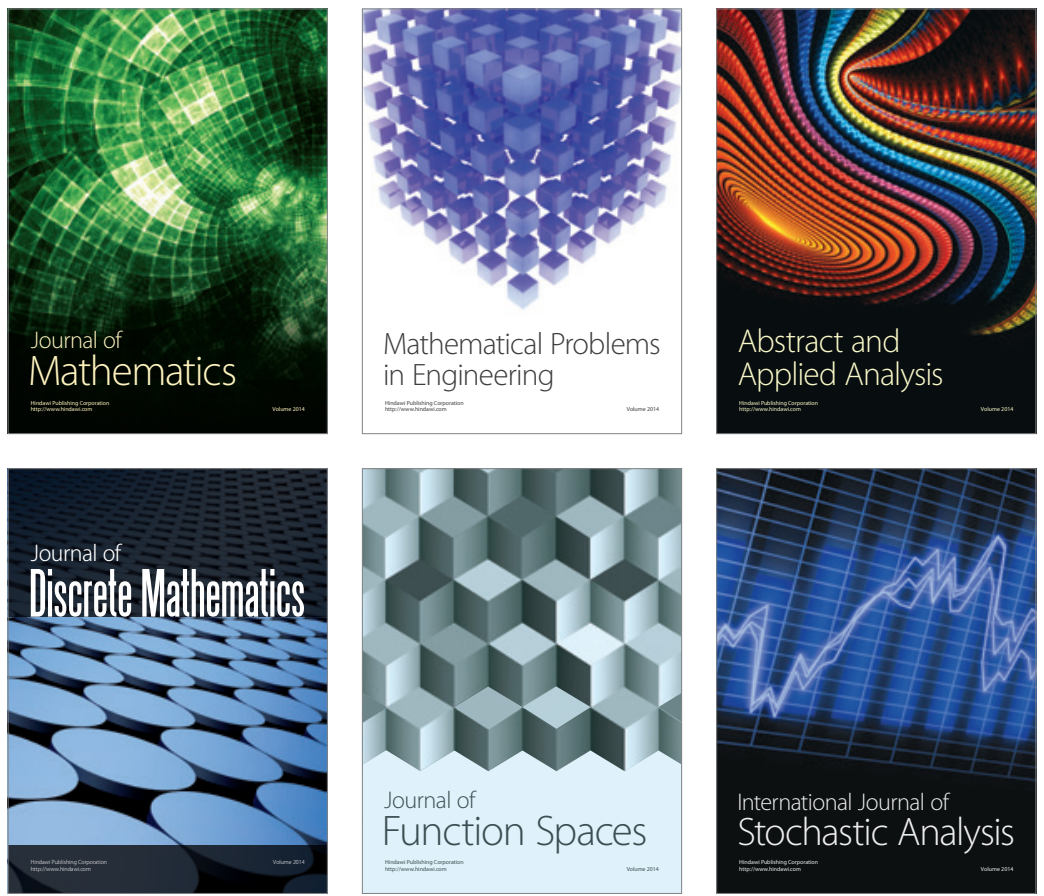

Journal of

Function Spaces

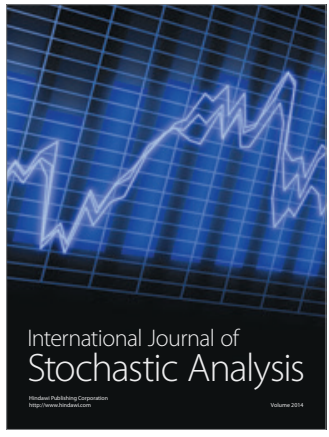

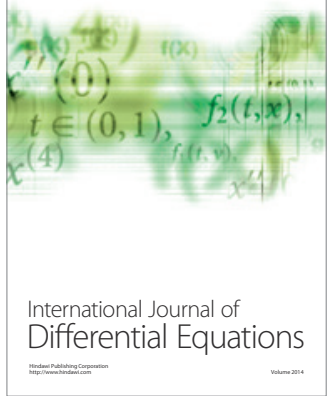
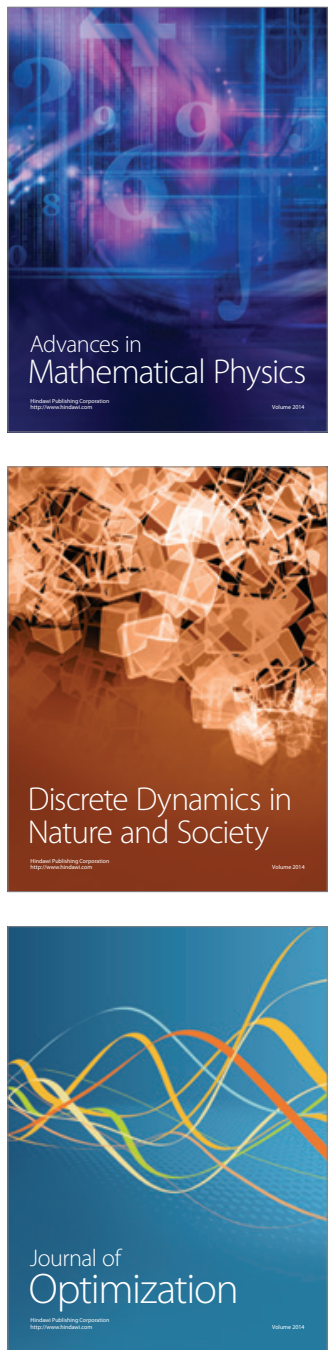